\title{
Critical exponents for the one-dimensional Hubbard model
}

\author{
Holger Frahm \\ Department of Physics, University of Virginia, Charlottesville, Virginia 22901 \\ V. E. Korepin \\ Institute for Theoretical Physics, State University of New York at Stony Brook, \\ Stony Brook, New York 11794-3840 \\ (Received 7 May 1990)
}

\begin{abstract}
Using results on the scaling of energies with the size of the system and the principles of conformal quantum field theory, we calculate the asymptotics of correlation functions for the one-dimensional Hubbard model in the repulsive regime in the presence of an external magnetic field. The critical exponents are given in terms of a dressed charge matrix that is defined in terms of a set of integral equations obtained from the Bethe-Ansatz solution for the Hubbard model. An interpretation of this matrix in terms of thermodynamical coefficients is given, and several limiting cases are considered.
\end{abstract}

\section{INTRODUCTION}

Despite remarkable efforts the understanding of the physics of correlated electron systems is still far from complete. Even in one spatial dimension where the Hubbard Hamiltonian provides the opportunity to study correlation effects in an integrable model, ${ }^{1-8}$ apparently simple quantities like the asymptotics of correlation functions have been calculated in certain limits only. ${ }^{7,9-11}$

The Hubbard model is only one of a large class of one-dimensional models with a critical point at $T=0$. For these systems the correlation functions decay asymptotically as powers of the distance. It is especially the calculation of the exponents characterizing this power-law behavior that have attracted longstanding theoretical interest. Considerable results in this field have been obtained from perturbational calculations and renormalization-group treatments in various models. ${ }^{9,12-14}$ Haldane realized that many critical onedimensional (1D) models can be described within the socalled Luttinger-liquid approach ${ }^{15-18}$-which in essence is based on the fact that they are certain realizations of the Gaussian model. ${ }^{19} \mathrm{He}$ also first calculated the corresponding coupling constant (which governs the critical behavior) from Bethe-Ansatz equations for models with a single degree of freedom. ${ }^{16}$ We would like to mention the quantum inverse scattering approach to correlation functions. ${ }^{20}$ This approach shows that the critical exponents of integrable models (i.e., the conformal dimensions of the fields) depend only on the underlying $R$ matrix that satisfies the Yang-Baxter relation. Hence every solution of this relation defines a large number of integrable systems that show the same universal critical behavior.

Recently, the advances in the understanding of critical phenomena in (1+1)-dimensional quantum systems as a consequence of conformal invariance ${ }^{21}$ have provided a great deal of new insight in the problems described above. This concept provides a simple means for the classification of universality classes in terms of a single dimensionless number, namely, the central charge $c$ of the underlying Virasoro algebra. The critical exponents in a conformally invariant theory are the scaling dimensions of the operators within this model and for a Gaussian theory (which corresponds to $c=1$ and is relevant to many one-dimensional critical systems) they are known to depend on the coupling constant of this theory only. Both the central charge $c$ and the operator dimensions can be calculated by considering the scaling behavior of the energies of the ground state and low-lying states as a function of the size of the system. ${ }^{22,23}$ In Bethe-Ansatz soluble systems these finite-size corrections can be evaluated exactly. ${ }^{24-31,6,8}$ As mentioned above the critical behavior of models with one degree of freedom (like bosons or spinless fermions) is described by a Virasoro algebra with central charge $c=1$ in the generic situation (i.e., at arbitrary chemical potential and, e.g., magnetic field).

Unfortunately, however, these concepts cannot be applied directly to the Hubbard model, since conformal quantum field theory deals with Lorentz-invariant systems only - i.e., it requires all gapless excitations to have the same velocity. This is not true in the Hubbard model: In the generic case there coexist charge- and spin-density waves with vanishing gap that have different velocities. Standard conformal-field-theory considerations apply only at half filling, where the charge-density waves develop a gap. Very recently, however, the investigation of the spectra of multicomponent integrable systems of finite-size has suggested a generalization of the relation between critical exponents and finite-size scaling amplitudes from the conformal case to models with more than one gapless excitation. ${ }^{29,8,30}$ These models with several degrees of freedom (e.g., fermions with spin) 
can be solved by a hierarchy of Bethe Ansätze. ${ }^{32}$ It is found that to each critical degree of freedom there corresponds one Virasoro algebra each of which is again characterized by a central charge $c=1$ in the generic case. The complete critical theory is given as a semidirect product of these algebras. The operator dimensions in these models are functions of the elements of a matrix of coupling constants. The asymptotics of correlation functions for models of this type have been obtained in Ref. 29 .

In this paper we want to apply these new ideas to the calculation of the long distance asymptotics of correlation functions in the spacelike regime for the one-dimensional Hubbard model. This model describes electrons with spin $\frac{1}{2}$ on the lattice. The electrons are described in terms of canonical Fermi fields $\psi_{j, \sigma}$ where $j$ is the number of the lattice site $(j=1, \ldots, N)$ and $\sigma=\uparrow, \downarrow$ is the spin. The $\psi_{j, \sigma}$ obey standard anticommutation relations: $\left\{\psi_{i, \sigma}^{\dagger}, \psi_{j, \sigma^{\prime}}^{\dagger}\right\}=\left\{\psi_{i, \sigma}, \psi_{j, \sigma^{\prime}}\right\}=0, \quad\left\{\psi_{i, \sigma}, \psi_{j, \sigma^{\prime}}^{\dagger}\right\}=\delta_{i j} \delta_{\sigma \sigma^{\prime}}$,

$\psi_{i, \sigma}|0\rangle=\langle 0| \psi_{i, \sigma}^{\dagger}=0$.

Here $|0\rangle$ and $\langle 0|$ are the Fock vacuum and the dual Fock vacuum, respectively.

The Hamiltonian for the one-dimensional Hubbard model is given by the following expression:

$$
\begin{aligned}
\mathcal{H}= & -\sum_{j=1}^{N} \sum_{\sigma}\left(\psi_{j+1, \sigma}^{\dagger} \psi_{j, \sigma}+\psi_{j, \sigma}^{\dagger} \psi_{j+1, \sigma}\right)+4 u \sum_{j=1}^{N} n_{j \uparrow} n_{j \downarrow} \\
& +\mu \sum_{j=1}^{N}\left(n_{j \uparrow}+n_{j \downarrow}\right)-\frac{h}{2} \sum_{j=1}^{N}\left(n_{j \uparrow}-n_{j \downarrow}\right)
\end{aligned}
$$

Here $n_{j, \sigma}=\psi_{j, \sigma}^{\dagger} \psi_{j, \sigma}$ is the number of spin $\sigma$ electrons at site $\mathrm{j}, 4 u>0$ is the on-site Coulomb repulsion, $\mu$ is the chemical potential, and $h$ is an external magnetic field.

For the attractive case $u<0$ the asymptotic behavior of correlation functions has been calculated in Ref. 7 . Here we shall study correlation functions for the repulsive case at both zero and small finite temperature.

Our paper is organized as follows: In the following section we review the Bethe-Ansatz solution for the Hubbard model and introduce the various quantities that will be relevant in the description of the critical behavior of the Hubbard model later. In Sec. III we summarize the consequences of conformal invariance regarding the scaling of the energies of the ground state and low-lying states with the size of the system and the relation to correlation functions of primary fields. Conformal quantum field theory deals with Lorentz-invariant systems at criticality. To apply these concepts to the present case of two kinds of gapless excitations with different velocities they have to be generalized. The proper generalization is suggested by the altered finite-size corrections in the spectrum. It will be shown that the critical behavior is described in terms of a $2 \times 2$ matrix rather than a scalar coupling constant as in the single-component case-this is the so-called dressed charge matrix. In Sec. IV we outline the procedure used to calculate the long-distance asymptotics for various correlation functions. In Secs. VVII we consider several limiting cases where the elements of the dressed charge matrix can be parametrized by a scalar quantity, which allows us to give explicit expressions for the critical exponents. These cases are the Hubbard model without magnetic field, in a magnetic field strong enough to yield maximal magnetization, andfor comparison with earlier results and to demonstrate the effect of an external magnetic field on the critical behavior-the Hubbard model at half filling. In addition we discuss how the dressed charge relates to physical quantities like magnetic susceptibility and compressibility of the system. Finally, in Sec. VIII we generalize these relations to the general case.

\section{THE BETHE-ANSATZ SOLUTION FOR THE HUBBARD MODEL}

Lieb and $\mathrm{Wu}^{1}$ have solved the Schrödinger equation for the Hubbard model (1.2) using the Bethe Ansatz. The eigenstates of the chain with $N_{c}=N_{\uparrow}+N_{\downarrow}$ electrons and $N_{s}=N_{\downarrow}$ down spins are characterized by the momenta $k_{j}$ of (spinless) charges (also called holons recently) and the rapidities $\lambda_{\alpha}$ of spin waves (spinons). Imposing periodic boundary conditions on the wave functions leads to the Bethe-Ansatz equations

$N k_{j}=2 \pi I_{j}+\sum_{\beta=1}^{N_{s}} 2 \arctan \left(\frac{\sin k_{j}-\lambda_{\beta}}{u}\right)$,

$$
\begin{aligned}
& \sum_{j=1}^{N_{c}} 2 \arctan \left(\frac{\lambda_{\alpha}-\sin k_{j}}{u}\right) \\
& \quad=2 \pi J_{\alpha}+\sum_{\beta=1}^{N_{s}} 2 \arctan \left(\frac{\lambda_{\alpha}-\lambda_{\beta}}{2 u}\right) .
\end{aligned}
$$

Here $N$ is the length of the system in units of the lattice spacing. The quantum numbers $I_{j}$ and $J_{\alpha}$ are integer or half-odd integer, depending on the parities of the numbers of down and up spins, respectively:

$$
I_{j}=\frac{N_{s}}{2} \bmod 1, J_{\alpha}=\frac{N_{c}-N_{s}+1}{2} \bmod 1 .
$$

The energy and momentum of the system in a state corresponding to a solution of (2.1) are equal to

$$
\begin{aligned}
& E=-2 \sum_{j=1}^{N_{c}} \cos k_{j}+\mu N_{c}+h\left(N_{s}-\frac{N_{c}}{2}\right), \\
& P=\sum_{j=1}^{N_{c}} k_{j}=\frac{2 \pi}{N}\left(\sum_{j} I_{j}+\sum_{\alpha} J_{\alpha}\right) .
\end{aligned}
$$


In the thermodynamic limit $\left(N \rightarrow \infty\right.$, with $N_{c} / N$, $N_{s} / N$ kept constant) the ground state of the model is a Fermi sea, characterized by two distribution functions: $\rho_{c}(k)$ is the distribution function of charges with momentum $k$, and $\rho_{s}(\lambda)$ describes the distribution of down spins with respect to the spin-wave rapidity $\lambda$. The functions $\rho_{c}(k)$ and $\rho_{s}(\lambda)$ satisfy the following integral equations:

$$
\begin{aligned}
\rho_{c}(k)= & \frac{1}{2 \pi}+\frac{\cos k}{2 \pi} \int_{-\lambda_{0}}^{\lambda_{0}} d \lambda K_{1}(\sin k-\lambda) \rho_{s}(\lambda) \\
\rho_{s}(\lambda)= & \frac{1}{2 \pi} \int_{-k_{0}}^{k_{0}} d k K_{1}(\lambda-\sin k) \rho_{c}(k) \\
& -\frac{1}{2 \pi} \int_{-\lambda_{0}}^{\lambda_{0}} d \mu K_{2}(\lambda-\mu) \rho_{s}(\mu)
\end{aligned}
$$

The kernels $K_{1}, K_{2}$ of these integral equations are given by

$$
K_{1}(x)=\frac{2 u}{u^{2}+x^{2}}, \quad K_{2}(x)=\frac{4 u}{4 u^{2}+x^{2}} .
$$

The values of $\lambda_{0}$ and $k_{0}$ are fixed by the following equations:

$$
\begin{aligned}
& \int_{-k_{0}}^{k_{0}} d k \rho_{c}(k)=\frac{N_{c}}{N} \equiv n_{c} \\
& \int_{-\lambda_{0}}^{\lambda_{0}} d \lambda \rho_{s}(\lambda)=\frac{N_{s}}{N} \equiv \frac{1}{2} n_{c}-\mathcal{M}
\end{aligned}
$$

In Eq. (2.6) $n_{c}$ is the total density of electrons and $\mathcal{M}$ $=\left(N_{\uparrow}-N_{\downarrow}\right) / 2 N$ is the magnetization per lattice site. The ground-state energy per lattice site is

$$
\begin{aligned}
\varepsilon_{0}= & \int_{-k_{0}}^{k_{0}} d k\left(\mu-\frac{h}{2}-2 \cos k\right) \rho_{c}(k) \\
& +h \int_{-\lambda_{0}}^{\lambda_{0}} d \lambda \rho_{s}(\lambda) .
\end{aligned}
$$

Alternatively, $\varepsilon_{0}$ may be given in terms of the dressed energy

$$
\varepsilon_{0}=\frac{1}{2 \pi} \int_{-k_{0}}^{k_{0}} d k \varepsilon_{c}(k),
$$

where $\varepsilon_{c}(k)$ is the solution of the system of coupled integral equations:

$$
\begin{aligned}
\varepsilon_{c}(k)= & \varepsilon_{c}^{(0)}(k)+\frac{1}{2 \pi} \int_{-\lambda_{0}}^{\lambda_{0}} d \lambda K_{1}(\sin k-\lambda) \varepsilon_{s}(\lambda) \\
\varepsilon_{s}(\lambda)= & \varepsilon_{s}^{(0)}(\lambda)+\frac{1}{2 \pi} \int_{-k_{0}}^{k_{0}} d k \cos k K_{1}(\lambda-\sin k) \varepsilon_{c}(k) \\
& -\frac{1}{2 \pi} \int_{-\lambda_{0}}^{\lambda_{0}} d \mu K_{2}(\lambda-\mu) \varepsilon_{s}(\mu)
\end{aligned}
$$

The bare energies $\varepsilon_{c, s}^{(0)}$ are obtained from (2.3) as

$$
\varepsilon_{c}^{(0)}(k)=\mu-\frac{h}{2}-2 \cos k, \quad \varepsilon_{s}^{(0)}(\lambda)=h .
$$

The solutions of Eqs. (2.9) define the energy bands. The ground-state configuration corresponds to the filling of all states with $\varepsilon_{c}(k)<0$ and $\varepsilon_{s}(\lambda)<0$. Consequently, the conditions

$$
\varepsilon_{c}\left(k_{0}\right)=0, \quad \varepsilon_{s}\left(\lambda_{0}\right)=0
$$

provide another way to define the values of $k_{0}$ and $\lambda_{0}$ for the ground state for given magnetic field $h$ and chemical potential $\mu$.

Another quantity that will be useful in the description of the critical behavior below is the dressed charge matrix: $29,8,30$

$$
\left(\begin{array}{ll}
\xi_{c c}(k) & \xi_{c s}(\lambda) \\
\xi_{s c}(k) & \xi_{s s}(\lambda)
\end{array}\right)
$$

(scalar versions of this quantity have been introduced before in Refs. 17, 20, 25, and 26), which is defined as the solution of the following integral equations:

$$
\begin{aligned}
\xi_{c c}(k)= & 1+\frac{1}{2 \pi} \int_{-\lambda_{0}}^{\lambda_{0}} d \lambda \xi_{c s}(\lambda) K_{1}(\lambda-\sin k) \\
\xi_{c s}(\lambda)= & \frac{1}{2 \pi} \int_{-k_{0}}^{k_{0}} d k \cos k \xi_{c c}(k) K_{1}(\sin k-\lambda) \\
& -\frac{1}{2 \pi} \int_{-\lambda_{0}}^{\lambda_{0}} d \mu \xi_{c s}(\mu) K_{2}(\mu-\lambda), \\
\xi_{s c}(k)= & \frac{1}{2 \pi} \int_{-\lambda_{0}}^{\lambda_{0}} d \lambda \xi_{s s}(\lambda) K_{1}(\lambda-\sin k), \\
\xi_{s s}(\lambda)= & 1+\frac{1}{2 \pi} \int_{-k_{0}}^{k_{0}} d k \cos k \xi_{s c}(k) K_{1}(\sin k-\lambda) \\
& -\frac{1}{2 \pi} \int_{-\lambda_{0}}^{\lambda_{0}} d \mu \xi_{s s}(\mu) K_{2}(\mu-\lambda) .
\end{aligned}
$$

(This definition of the dressed charge matrix is the one ised in Refs. 29 and 30, and Woynarovich ${ }^{8}$ uses its transose.)

The spectrum of conformal dimensions and critical exponents will be expressed in terms of the elements of this matrix at $k=k_{0}$ and $\lambda=\lambda_{0}$. In Sec. VIII we shall relate these quantities to certain thermodynamic coefficients.

\section{CONFORMAL QUANTUM-FIELD- THEORY APPROACH FOR CORRELATION FUNCTIONS}

The Hubbard model is critical at zero temperature. Recently, much progress in the description of critical phenomena has been made by application of the concepts of conformal quantum field theory. ${ }^{21}$ Due to conformal invariance, the universality class of the model under consideration is uniquely described by a single dimensionless 
number $c$ - the central charge of the underlying Virasoro algebra. The value of $c$ can be extracted from the finitesize-scaling behavior of the ground-state energy 22,23

$$
E_{0}-N \varepsilon_{0}=-\frac{\pi}{6 N} v c+O\left(\frac{1}{N}\right) \text {. }
$$

Here $N$ is the size of the system, $E_{0}$ is the ground-state energy of the finite system, $\varepsilon_{0}$ is the energy density of the ground state of the infinite system, and $v$ is the Fermi velocity.

Each primary field $\phi_{\Delta^{ \pm}}$with scaling dimension $x$ $=\Delta^{+}+\Delta^{-}$and spin $s=\Delta^{+}-\Delta^{-}$gives rise to a tower of excited states. The energies and momenta of these excitations scale as $^{22}$

$$
\begin{aligned}
& E_{\Delta^{ \pm}}^{N^{+}, N^{-}}-E_{0}=\frac{2 \pi v}{N}\left(x+N^{+}+N^{-}\right)+O\left(\frac{1}{N}\right) \\
& P_{\Delta^{ \pm}}^{N^{+}, N^{-}}-P_{0}=\frac{2 \pi}{N}\left(s+N^{+}-N^{-}\right)+2 D \mathcal{P}_{F}
\end{aligned}
$$

Here $N^{+}, N^{-}$are positive integers (enumerating the descendent fields), and $2 D$ is the momentum of the state in units of the Fermi momentum $\mathcal{P}_{F}$. The correlation functions of the primary fields are known to be $\mathrm{e}^{21,25}$

$$
\left\langle\phi_{\Delta^{ \pm}}(x, t) \phi_{\Delta^{ \pm}}(0,0)\right\rangle=\frac{\exp \left(-2 i D \mathcal{P}_{F} x\right)}{(x-i v t)^{2 \Delta^{+}}(x+i v t)^{2 \Delta^{-}}}
$$

In the following we want to apply these concepts to calculate the asymptotics of correlation functions for the Hubbard model. In the repulsive case that we are considering here we have to distinguish two different situations.

(a) For a half-filled band, $n_{c}=1$, only the spin excitations are gapless. From finite-size calculations ${ }^{6}$ the corresponding continuum field theory is known to be a Gaussian model ${ }^{19}$ with central charge $c=1$ and the dimensions of the primary operators are given by

$$
\Delta^{ \pm}\left(\Delta N_{s}, D_{s}\right)=\frac{1}{2}\left(D_{s} X \pm \frac{\Delta N_{s}}{2 X}\right)^{2}
$$

the dimensions of descendent fields differ from $\Delta^{ \pm}$by the integers $N^{ \pm}$. The coupling constant $X$ of this Gaussian model depends on the system parameters - an explicit expression is given in Sec. VII.

(b) In the case of less than half filling, $n_{c}=1-\delta$, where $\delta>0$ is the doping, both charge and spin excitations are gapless. Here the finite-size corrections are not of the form of (3.1) and (3.2) but rather ${ }^{8}$

$$
E_{0}-N \varepsilon_{0}=-\frac{\pi}{6 N}\left(v_{c}+v_{s}\right)+O\left(\frac{1}{N}\right)
$$

and

$$
\begin{aligned}
& E(\Delta \mathbf{N}, \mathbf{D})-E_{0}=\frac{2 \pi}{N}\left[\frac{1}{4} \Delta \mathbf{N}^{T}\left(Z^{-1}\right)^{T} V Z^{-1} \Delta \mathbf{N}+\mathbf{D}^{T} Z V Z^{T} \mathbf{D}+v_{c}\left(N_{c}^{+}+N_{c}^{-}\right)+v_{s}\left(N_{s}^{+}+N_{s}^{-}\right)\right]+O\left(\frac{1}{N}\right) \\
& P(\Delta \mathbf{N}, \mathbf{D})-P_{0}=\frac{2 \pi}{N}\left(\Delta \mathbf{N}^{T} \cdot \mathbf{D}+N_{c}^{+}-N_{c}^{-}+N_{s}^{+}-N_{s}^{-}\right)+2 D_{c} \mathcal{P}_{F, \uparrow}+2\left(D_{c}+D_{s}\right) \mathcal{P}_{F, \downarrow} \\
& V=\operatorname{diag}\left(v_{c}, v_{s}\right) .
\end{aligned}
$$

(Similar expressions have been found in different models in Refs. 29 and 30.) For the Hubbard model, $P_{0}=0$. Here $\Delta \mathbf{N}$ and $\mathbf{D}$ are vectors characterizing the excited state under consideration, $N_{c}^{ \pm}$and $N_{s}^{ \pm}$are positive integers, and $v_{c}$ and $v_{s}$ are the Fermi velocities of charge and spin density waves, respectively:

$$
v_{c}=\frac{1}{2 \pi \rho_{c}\left(k_{0}\right)} \varepsilon_{c}^{\prime}\left(k_{0}\right), \quad v_{s}=\frac{1}{2 \pi \rho_{s}\left(\lambda_{0}\right)} \varepsilon_{s}^{\prime}\left(\lambda_{0}\right) \text {. }
$$

$\mathcal{P}_{F, \uparrow}$ and $\mathcal{P}_{F, \downarrow}$ are the Fermi momenta for electrons with spin up and down, respectively, i.e.,

$$
\mathcal{P}_{F, \uparrow(\downarrow)}=\frac{1}{2}\left(\pi n_{c} \pm 2 \pi \mathcal{M}\right)
$$

( $\mathcal{M}$ is the magnetization of the system). $Z$ is a $2 \times 2$ matrix related to the dressed charge (2.12) in the following way:

$$
Z \equiv\left(\begin{array}{cc}
Z_{c c} & Z_{c s} \\
Z_{s c} & Z_{s s}
\end{array}\right)=\left(\begin{array}{l}
\xi_{c c}\left(k_{0}\right) \xi_{c s}\left(\lambda_{0}\right) \\
\xi_{s c}\left(k_{0}\right) \xi_{s s}\left(\lambda_{0}\right)
\end{array}\right)
$$

Equations (3.5) and (3.6) can be interpreted in terms of a semidirect product of two independent Virasoro algebras, both having central charge equal to one. ${ }^{29}$

The elements of $\Delta \mathbf{N}$ and $\mathbf{D}$ depend on certain commensuration conditions for $N_{c}, N_{s}$ and the size $N$ of the system. ${ }^{8}$ In the thermodynamic limit the ground state is unique with $\Delta N_{c}=\Delta N_{s}=D_{c}=D_{s}=0$. For excited states, $\Delta N$ has integer components denoting the change of the numbers of electrons and down spins with respect to the ground state. Due to the restrictions $(2.2), D_{c}$ and $D_{s}$ are integers or half-odd integers depending on the parities of $\Delta N_{c}$ and $\Delta N_{s}$ :

$$
D_{c}=\frac{\Delta N_{c}+\Delta N_{s}}{2} \bmod 1, \quad D_{s}=\frac{\Delta N_{c}}{2} \bmod 1 .
$$

To find the analog of Eq. (3.3) for the correlation func- 
tions of primary fields for this case we rewrite Eqs. (3.6) as

$$
\begin{aligned}
E(\Delta \mathbf{N}, \mathbf{D})-E_{0}= & \frac{2 \pi}{N}\left[v_{c}\left(\Delta_{c}^{+}+\Delta_{c}^{-}\right)+v_{s}\left(\Delta_{s}^{+}+\Delta_{s}^{-}\right)\right] \\
& +O\left(\frac{1}{N}\right)
\end{aligned}
$$$$
\begin{aligned}
P(\Delta \mathbf{N}, \mathbf{D})-P_{0}= & \frac{2 \pi}{N}\left(\Delta_{c}^{+}-\Delta_{c}^{-}+\Delta_{s}^{+}-\Delta_{s}^{-}\right) \\
& +2 D_{c} \mathcal{P}_{F, \uparrow}+2\left(D_{c}+D_{s}\right) \mathcal{P}_{F, \downarrow}
\end{aligned}
$$

Comparing Eqs. (3.6) and (3.11) we obtain expressions for the conformal dimensions of the fields in this theory as functions of the elements of the dressed charge matrix (3.9). Note that the gap in the momentum (3.11) does not fix the conformal spins $s_{c, s}=\Delta_{c, s}^{+}-\Delta_{c, s}^{-}$uniquely. In addition we have to require that all the dimensions $\Delta_{c, s}^{ \pm}$be nonnegative. (Otherwise there would be unphysical divergences in the correlation functions.) As in the scalar case (3.4) this can be guaranteed by writing the dimensions as complete squares:

$$
\begin{aligned}
2 \Delta_{c}^{ \pm}(\Delta \mathbf{N}, \mathbf{D})= & \left(Z_{c c} D_{c}+Z_{s c} D_{s}\right. \\
& \left. \pm \frac{Z_{s s} \Delta N_{c}-Z_{c s} \Delta N_{s}}{2 \operatorname{det} Z}\right)^{2}+2 N_{c}^{ \pm} \\
2 \Delta_{s}^{ \pm}(\Delta \mathbf{N}, \mathbf{D})= & \left(Z_{c s} D_{c}+Z_{s s} D_{s}\right. \\
& \left. \pm \frac{Z_{c c} \Delta N_{s}-Z_{s c} \Delta N_{c}}{2 \operatorname{det} Z}\right)^{2}+2 N_{s}^{ \pm}
\end{aligned}
$$

In general, the conformal spins defined by these expressions will depend on the system parameters. In absence of a magnetic field, however, they are found to be functions of the numbers $\Delta N_{c, s}$ and $D_{c, s}$ only (see Sec. $\mathrm{V}$ below).

Generalizing the expression (3.3) for the scalar case, we can write down the correlation functions for primary fields in the present theory as

$$
\left\langle\phi_{\Delta^{ \pm}}(x, t) \phi_{\Delta^{ \pm}}(0,0)\right\rangle=\frac{\exp \left(-2 i D_{c} \mathcal{P}_{F, \uparrow} x\right) \exp \left[-2 i\left(D_{c}+D_{s}\right) \mathcal{P}_{F, \downarrow} x\right]}{\left(x-i v_{c} t\right)^{2 \Delta_{c}^{+}}\left(x+i v_{c} t\right)^{2 \Delta_{c}^{-}}\left(x-i v_{s} t\right)^{2 \Delta_{s}^{+}}\left(x+i v_{s} t\right)^{2 \Delta_{s}^{-}}}
$$

The correlation functions of the physical fields in the Hubbard model will consist of a sum of terms (3.13). ${ }^{29}$

At small finite temperature $T>0$ the correlation functions decay exponentially, however with a small exponent because of the vicinity of the phase transition. Hence, the long-distance asymptotics of correlation functions in the spacelike regime can still be obtained from conformal invariance. In this case expressions of type (3.13) have to be replaced by

$$
\begin{aligned}
\exp \left(-2 i D_{c} \mathcal{P}_{F, \uparrow} x\right) \exp \left[-2 i\left(D_{c}+D_{s}\right) \mathcal{P}_{F, \downarrow} x\right] & \left(\frac{\pi T}{v_{c} \sinh \left[\pi T\left(x-i v_{c} t\right) / v_{c}\right]}\right)^{2 \Delta_{c}^{+}}\left(\frac{\pi T}{v_{c} \sinh \left[\pi T\left(x+i v_{c} t\right) / v_{c}\right]}\right)^{2 \Delta_{c}^{-}} \\
& \times\left(\frac{\pi T}{v_{s} \sinh \left[\pi T\left(x-i v_{s} t\right) / v_{s}\right]}\right)^{2 \Delta_{s}^{+}}\left(\frac{\pi T}{v_{s} \sinh \left[\pi T\left(x-i v_{s} t\right) / v_{s}\right]}\right)^{2 \Delta_{s}^{-}}
\end{aligned}
$$

This expression can be obtained from (3.13) by conformal mapping of the complex plane without the origin (zero temperature) onto a strip of width $1 / T$ in time direction.

\section{ASYMPTOTIC EXPRESSIONS FOR CORRELATION FUNCTIONS}

In this section we want to calculate asymptotic expressions for correlation functions of certain physical operators using the predictions of conformal quantum field theory outlined above. In particular, we want to consider the field correlator

$$
G_{\psi \psi}(x, t)=\left\langle\psi_{\downarrow}(x, t) \psi_{\downarrow}^{\dagger}(0,0)\right\rangle .
$$

The density-density correlator is given by

$$
\begin{aligned}
& G_{n n}(x, t)=\langle n(x, t) n(0,0)\rangle, \\
& n(x, t)=n_{\uparrow}(x, t)+n_{\downarrow}(x, t) .
\end{aligned}
$$

The spin-spin correlation functions are defined by

$$
\begin{aligned}
& G_{\sigma \sigma}^{z}(x, t)=\left\langle S^{z}(x, t) S^{z}(0,0)\right\rangle, \\
& G_{\sigma \sigma}^{\perp}(x, t)=\left\langle S^{-}(x, t) S^{+}(0,0)\right\rangle,
\end{aligned}
$$

where $S^{z}(x, t)=\left[n_{\uparrow}(x, t)-n_{\downarrow}(x, t)\right] / 2$ and $S^{+}(x, t)$ $=\psi_{\uparrow}^{\dagger}(x, t) \psi_{\downarrow}(x, t)$.

Finally, we want to consider the correlation functions for singlet and triplet pairs

$$
\begin{aligned}
& G_{p}^{(0)}(x, t)=\left\langle\psi_{\uparrow}^{\dagger}(x, t) \psi_{\downarrow}^{\dagger}(x, t) \psi_{\downarrow}(0,0) \psi_{\uparrow}(0,0)\right\rangle, \\
& G_{p}^{(1)}(x, t)=\left\langle\psi_{\downarrow}^{\dagger}(x+1, t) \psi_{\downarrow}^{\dagger}(x, t) \psi_{\downarrow}(1,0) \psi_{\downarrow}(0,0)\right\rangle .
\end{aligned}
$$

All of these correlators are of the form $\left\langle\mathcal{O}(x, t) \mathcal{O}^{\dagger}(0,0)\right\rangle$. To find their asymptotic behavior one has to expand the operator $\mathcal{O}$ in terms of the conformal fields. Usually, 
this is not possible (for the nonlinear Schrödinger model this expansion has been performed in Ref. 28). However, since $\mathcal{O}$ is known in terms of electron creation and annihilation operators the quantum numbers $N_{c}$ and $N_{s}$ of the intermediate states are known. Hence, in a generic situation the leading term in the asymptotic expansion of the correlation functions decays with critical exponents obtained from (3.12) with the corresponding values of $\Delta N_{c}$ and $\Delta N_{s}$ by minimizing with respect to $D_{c}, D_{s}$ using the restrictions (3.10). At special points in the phase diagram, however, the corresponding amplitude may vanish (this happens, for example, in the case of the $2 \mathcal{P}_{F}$ singularity of the density-density correlator at $u=\infty$ and $h=0$, see Sec. V B below).

As mentioned above, the spectrum of charged excitations has a gap at half filling $n_{c}=1$. Hence, the correla- tion functions (4.1), (4.4), and (4.5) will decay exponentially. The density-density (4.2) and spin-spin correlators (4.3), however, still decay as powers of the distance (see Sec. VII below).

As an illustration of this scheme let us describe how to obtain the asymptotical behavior of the density-density correlations $G_{n n}(x, t)(4.2)$ in the Euclidean region: The leading term is a constant, since

$$
\langle n(x, t)\rangle=n_{c}
$$

To write down the higher terms we have to use (3.12) and (3.13) at $\Delta N_{c}=\Delta N_{s}=0$; the restrictions of Eqs. (3.10) are satisfied for any integer $D_{c}, D_{s}$. Hence the next terms in the asymptotic expansion of $G_{n n}$ are found to be

$$
\begin{aligned}
G_{n n}(x, t)-n_{c}^{2} \sim & A_{1} \cos \left(2 \mathcal{P}_{F, \uparrow} x+\varphi_{1}\right) /\left(\left|x+i v_{c} t\right|^{2\left(Z_{c c}-Z_{s c}\right)^{2}}\left|x+i v_{s} t\right|^{2\left(Z_{c s}-Z_{s s}\right)^{2}}\right) \\
& +A_{2} \cos \left(2 \mathcal{P}_{F, \downarrow} x+\varphi_{2}\right) /\left(\left|x+i v_{c} t\right|^{2 Z_{s c}^{2}}\left|x+i v_{s} t\right|^{2 Z_{s s}^{2}}\right) \\
& +A_{3} \cos \left[2\left(\mathcal{P}_{F, \uparrow}+\mathcal{P}_{F, \downarrow}\right) x+\varphi_{3}\right] /\left(\left|x+i v_{c} t\right|^{2 Z_{c c}^{2}}\left|x+i v_{s} t\right|^{2 Z_{c s}^{2}}\right) \\
& +A_{4} \frac{x^{2}-\left(v_{c} t\right)^{2}}{\left[x^{2}+\left(v_{c} t\right)^{2}\right]^{2}}+A_{5} \frac{x^{2}-\left(v_{s} t\right)^{2}}{\left[x^{2}+\left(v_{s} t\right)^{2}\right]^{2}}
\end{aligned}
$$

Here $A_{k}$ are constant coefficients, $\varphi_{k}$ unknown phases. The general expression reads

$$
G_{n n}(x, t)=\sum A\left(D_{c}, D_{s}, N_{c}^{ \pm}, N_{s}^{ \pm}\right) \frac{\exp \left(-2 i D_{c} \mathcal{P}_{F, \uparrow} x\right) \exp \left[-2 i\left(D_{c}+D_{s}\right) \mathcal{P}_{F, \downarrow} x\right]}{\left(x-i v_{c} t\right)^{2 \Delta_{c}^{+}}\left(x+i v_{c} t\right)^{2 \Delta_{c}^{-}}\left(x-i v_{s} t\right)^{2 \Delta_{s}^{+}}\left(x+i v_{s} t\right)^{2 \Delta_{s}^{-}}}
$$

where the sum runs over all integers $D_{c}, D_{s}$ and nonnegative integers $N_{c}^{ \pm}$and $N_{s}^{ \pm}$and

$$
\begin{aligned}
& \Delta_{c}^{ \pm}\left(\mathbf{D}, \mathbf{N}^{ \pm}\right)=\frac{1}{2}\left(Z_{c c} D_{c}+Z_{s c} D_{s}\right)^{2}+N_{c}^{ \pm}, \\
& \Delta_{s}^{ \pm}(\Delta \mathbf{N}, \mathbf{D})=\frac{1}{2}\left(Z_{c s} D_{c}+Z_{s s} D_{s}\right)^{2}+N_{s}^{ \pm} .
\end{aligned}
$$

At finite temperature the inverse powers in (4.8) have to be replaced by inverse hyperbolic functions as in (3.14).

Similarly, the asymptotics of the other correlation functions can be found with

$$
\begin{array}{lc}
G_{\psi \psi}: \quad \Delta N_{c}=1, \quad \Delta N_{s}=1, \\
D_{c}=0, \pm 1, \pm 2, \ldots, & D_{s}= \pm \frac{1}{2}, \pm \frac{3}{2}, \ldots, \\
G_{\sigma \sigma}^{\perp}: \Delta N_{c}=0, & \Delta N_{s}=1, \\
D_{c}= \pm \frac{1}{2}, \pm \frac{3}{2}, \ldots, & D_{s}=0, \pm 1, \pm 2, \ldots, \\
G_{p}^{(0)}: \quad \Delta N_{c}=2, & \Delta N_{s}=1, \\
D_{c}= \pm \frac{1}{2}, \pm \frac{3}{2}, \ldots, & D_{s}=0, \pm 1, \pm 2, \ldots, \\
G_{p}^{(1)}: \quad \Delta N_{c}=2, & \Delta N_{s}=2, \\
D_{c}=0, \pm 1, \pm 2, \ldots, & D_{s}=0, \pm 1, \pm 2, \ldots .
\end{array}
$$

The longitudinal spin-spin correlations $G_{\sigma \sigma}^{z}$ are of the same form as the density-density correlations (4.7) with different parameters $A_{k}$ and $\varphi_{k}$ and the constant term replaced by $\mathcal{M}^{2}$.

The presence of marginal operators in the theory can lead to logarithmic corrections to the conformal predictions for correlation functions. ${ }^{9,33}$ In the spectrum they are manifest through finite-size effects of order $1 /(N \ln N)$ in (3.1) and (3.2). ${ }^{22}$ These can also be calculated explicitly from the Bethe-Ansatz equations (see, e.g., Ref. 6).

The procedure outlined here gives the critical exponents as functions of the entries of the matrix $Z$ (3.9). In Sec. VIII we shall express this matrix in terms of observable quantities. Before that, however, we want to consider certain special cases where the equation (2.13) for the dressed charge matrix simplifies. This allows us to write down the critical exponents connected to the correlation functions (4.1)-(4.5) explicitly.

\section{ZERO MAGNETIC FIELD}

For vanishing magnetic field the ground state of the Hubbard model has magnetization $\mathcal{M}=0$ corresponding to $\lambda_{0}=\infty$. The systems of integral Eqs. (2.4), (2.9), and (2.13) can be reduced to scalar ones by Fourier transformation with respect to $\lambda$. In particular we obtain 


$$
\begin{aligned}
\rho_{c}(k)= & \frac{1}{2 \pi}+\frac{\cos k}{2 \pi} \int_{-k_{0}}^{k_{0}} d k^{\prime} \bar{K}\left(\sin k-\sin k^{\prime}\right) \rho_{c}\left(k^{\prime}\right) \\
\varepsilon_{c}(k)= & \mu-2 \cos k \\
& +\frac{1}{2 \pi} \int_{-k_{0}}^{k_{0}} d k^{\prime} \cos k^{\prime} \bar{K}\left(\sin k-\sin k^{\prime}\right) \varepsilon_{c}\left(k^{\prime}\right), \\
\xi(k)=1 & +\frac{1}{2 \pi} \int_{-k_{0}}^{k_{0}} d k^{\prime} \cos k^{\prime} \bar{K}\left(\sin k-\sin k^{\prime}\right) \xi\left(k^{\prime}\right) \\
\xi_{c c}(k)= & \xi(k), \quad \xi_{s c}(k)=\frac{1}{2} \xi(k) .
\end{aligned}
$$

The kernel $\bar{K}(x)$ in these equations is

$$
\bar{K}(x)=\int_{0}^{\infty} d \omega \frac{e^{-u \omega}}{\cosh (u \omega)} \cos \omega x .
$$

The matrix $Z$ (3.9) has been calculated using WienerHopf techniques ${ }^{8}$

$$
Z=\left(\begin{array}{cc}
\xi\left(k_{0}\right) & 0 \\
\frac{1}{2} \xi\left(k_{0}\right) & \frac{1}{2} \sqrt{2}
\end{array}\right) .
$$

Rewriting the integral equation for $\xi$ as

$$
\begin{aligned}
\xi(z)=1+\frac{1}{2 \pi} \int_{-z_{0}}^{z_{0}} d z^{\prime} \int_{0}^{\infty} & d \omega \frac{e^{-\omega}}{\cosh (\omega)} \\
& \times \cos \left[\omega\left(z-z^{\prime}\right)\right] \xi\left(z^{\prime}\right),
\end{aligned}
$$

where $z=\sin k / u$, it becomes clear that the entries of the matrix $Z$ in (5.3) depend on $z_{0}=\sin k_{0} / u$ only.

Equation (5.4) can be solved in the limiting cases $u \gg \sin k_{0}$ and $0<u \ll \sin k_{0}$.

(a) For large $u / \sin k_{0}$ we find

$$
\xi\left(z_{0}\right)=1+\frac{\sin k_{0}}{\pi u} \ln 2 .
$$

This result allows us to write down the dressed charge at fixed $u$ for small densities

$$
\xi\left(z_{0}\right)=1+\frac{\ln 2}{u} n_{c}, \quad n_{c} \ll \min (1, u)
$$

and densities close to half filling $n_{c}=1-\delta(\delta$ is the doping):

$$
\xi\left(z_{0}\right)=1+\frac{\ln 2}{u f(u)} \delta, \quad \delta \ll u f(u),
$$

where ( $J_{0}$ is a Bessel function)

$$
\begin{aligned}
f(u) & =1-\int_{0}^{\infty} d \omega \frac{e^{-u \omega}}{\cosh (u \omega)} J_{0}(\omega) \\
& =1-2 \sum_{k=1}^{\infty} \frac{(-1)^{k+1}}{\sqrt{1+(2 u k)^{2}}} .
\end{aligned}
$$

Note that $f(u=0)=0$ but already $f(u=0.2)<2$ $\times 10^{-3}$. This indicates that near half filling and for $u<0.2$ the critical behavior is characterized by $\xi\left(z_{0}\right)$
$=\sqrt{2}[1-O(u)]$ for very small doping already.

For $u \gg 1$ we obtain $\xi$ for arbitrary values of the density explicitly

$$
\xi\left(z_{0}\right)=1+\frac{\ln 2}{\pi u} \sin \pi n_{c}, \quad u \gg 1 .
$$

(b) For small $u / \sin k_{0}$ we use a perturbative scheme ${ }^{34}$ based on the Wiener-Hopf method to solve Eq. (5.4) and obtain

$$
\xi\left(z_{0}\right)=\sqrt{2}\left(1-\frac{u}{2 \pi \sin k_{0}}\right) .
$$

For arbitrary repulsion $u>0$ and density $0<n_{c}<1$ the dressed charge $\xi\left(z_{0}\right)$ varies in the interval

$$
1 \leq \xi\left(z_{0}\right) \leq \sqrt{2} \text {. }
$$

We have solved the integral equation (5.1) for the $\rho_{c}(k)$ numerically for different values of $u$ and $k_{0}$. With these results we can plot the lines of constant $\xi\left(k_{0}\right)$ in the $n_{c}-u$ plane (see Fig. 1). Note that the critical exponents are functions of $\xi\left(k_{0}\right)$ only and consequently do not change along these lines. It can be seen clearly that the value of the total density where the dressed charge $\xi\left(k_{0}\right)$ takes its maximal value for given value of $u$ (this corresponds to $\left.k_{0}=\pi / 2\right)$ is always $n_{c}\left(k_{0}=\pi / 2\right)>\frac{1}{2}$ and goes to 1 as $u \rightarrow 0$.

\section{A. Relation of $\xi\left(k_{0}\right)$ to physical quantities}

Let us now discuss briefly the physical meaning of the quantity $\xi\left(z_{0}\right)$.

Following Refs. 16, 20, 8 , and 30 one can prove the identity

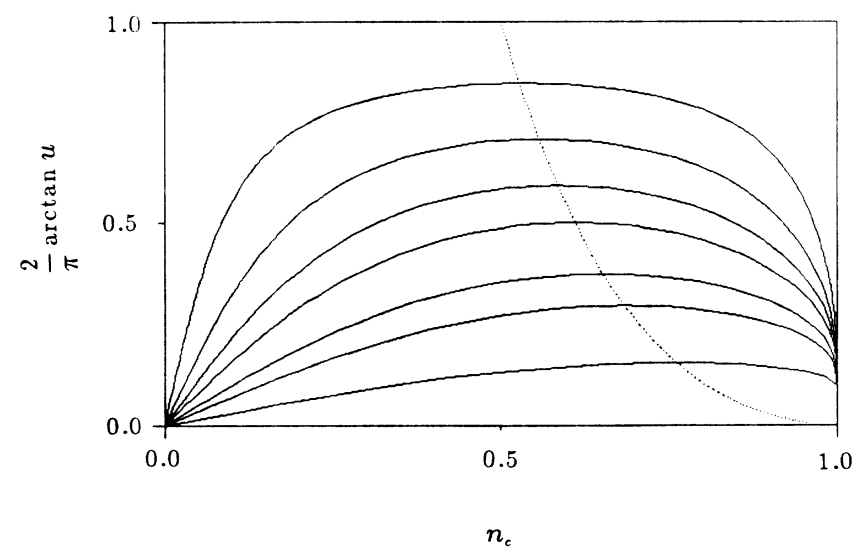

FIG. 1. Lines of constant universal behavior at zero magnetic field. Shown are the contours of constant dressed charge $\xi\left(k_{0}\right)$ [Eq. (5.3)] in the $n_{c^{-}} u$ plane: $\xi\left(k_{0}\right) \rightarrow 1(\sqrt{2})$ for $u \rightarrow \infty(0)$. The dotted line denotes the value of $n_{c}\left(k_{0}=\pi / 2\right)$ where the dressed charge takes its maximum value for given $u$. Note that the line $n_{c}=1$ is excluded here. There the Hubbard model falls into a different universality class-that of the isotropic Heisenberg antiferromagnet (see Sec. VII). 


$$
\frac{1}{2 \rho_{c}\left(k_{0}\right)} \frac{\partial n_{c}}{\partial k_{0}}=\xi\left(k_{0}\right)
$$

This relation clearly shows that the dressed charge reveals itself in a characteristic way in the number $2 \rho_{c}\left(k_{0}\right) \partial k_{0}$ of electrons added near the Fermi surface due to a change in the total density $n_{c}$.

A more physical interpretation of the dressed charge (which might even be relevant in non-Bethe-Ansatz integrable models) is found in its relation to certain thermodynamic coefficients ${ }^{17,20}$-in this case the compressibility of the electron gas ( $p$ is the pressure)

$$
\kappa=-\frac{1}{N} \frac{\partial N}{\partial p}=-\frac{N}{N_{c}^{2}} \frac{\partial N_{c}}{\partial \mu} .
$$

To see this consider the finite-size corrections (3.11). With Eq. (5.3) for the dressed charge matrix at vanishing magnetic field the expressions (3.12) for the conformal dimensions simplify to

$$
\begin{aligned}
\Delta_{c}^{ \pm}= & \frac{1}{2} \xi^{2}\left(D_{c}+\frac{1}{2} D_{s}\right)^{2}+\frac{1}{8 \xi^{2}}\left(\Delta N_{c}\right)^{2} \\
& \pm \frac{1}{4} \Delta N_{c}\left(2 D_{c}+D_{s}\right)+N_{c}^{ \pm}, \\
\Delta_{s}^{ \pm}= & \frac{1}{4}\left(D_{s}\right)^{2}+\frac{1}{4}\left(\Delta N_{s}-\frac{1}{2} \Delta N_{c}\right)^{2} \\
& \pm \frac{1}{4}\left(2 \Delta N_{s}-\Delta N_{c}\right) D_{s}+N_{s}^{ \pm} .
\end{aligned}
$$

(Note that the conformal spins $s_{c, s}=\Delta_{c, s}^{+}-\Delta_{c, s}^{-}$are independent of $\xi$.)

Now let us change the ground-state configuration under consideration by adding electrons while keeping zero magnetization, i.e., $\Delta N_{c}=2 \Delta N_{s}$ and $D_{c}=D_{s}=0$.
This state is actually the ground state of the system with $N_{c}+\Delta N_{c}$ electrons. From (3.11) we find the change in the ground-state energy due to this change of the electron number to be

$$
\Delta E=\frac{\pi v_{c}}{2 N} \frac{1}{\xi\left(k_{0}\right)^{2}}\left(\Delta N_{c}\right)^{2}
$$

To express this change in terms of the compressibility (5.13) we first have to separate the energy in the contribution of a microcanonical ensemble and the contribution of the chemical potential $E=\tilde{E}+\mu N_{c}$. Expansion of this quantity in powers of $\Delta N_{c}$ and comparison to (5.15) yields the thermodynamical relation $\mu=-\partial \tilde{E} / \partial N_{c}$ and the desired relation between the dressed charge and the compressibility:

$$
\xi\left(k_{0}\right)^{2}=\pi v_{c} n_{c}^{2} \kappa .
$$

An equivalent expression has been established for the 1D Bose gas with $\delta$ repulsion. ${ }^{17}$

\section{B. Correlation functions at zero magnetic field}

In the remainder of this section let us write down explicit expressions for the correlation functions (4.1)-(4.5). Note that the magnetization $\mathcal{M}$ of the system is zero, and hence $\mathcal{P}_{F, \uparrow}=\mathcal{P}_{F, \downarrow}=\pi n_{c} / 2 \equiv \mathcal{P}_{F}$. Below we give the critical exponents as functions of $\theta=2 \xi\left(k_{0}\right)^{2}$. Remember that according to Eq. (5.11) for any density $0<n_{c}<1$ the value of $\theta$ increases from 2 to 4 as the Coulomb repulsion $u$ decreases from $\infty$ to 0 . Following the scheme outlined in Sec. IV we obtain for the field correlator

$$
\begin{aligned}
G_{\psi \psi}(x, t) \sim & \frac{1}{\left|x+i v_{c} t\right|^{\nu_{1}}\left|x+i v_{s} t\right|^{1 / 2}} \operatorname{Re}\left[A e^{-i \mathcal{P}_{F} x}\left(\frac{x+i v_{c} t}{x-i v_{c} t}\right)^{1 / 4}\left(\frac{x+i v_{s} t}{x-i v_{s} t}\right)^{1 / 4}\right] \\
& +\frac{1}{\left|x+i v_{c} t\right|^{\nu_{3}}\left|x+i v_{s} t\right|^{1 / 2}} \operatorname{Re}\left[B e^{-3 i \mathcal{P}_{F} x}\left(\frac{x+i v_{c} t}{x-i v_{c} t}\right)^{3 / 4}\left(\frac{x-i v_{s} t}{x+i v_{s} t}\right)^{1 / 4}\right]
\end{aligned}
$$

where the exponent $\nu_{1}=1 / \theta+\theta / 16$ is a monotonically growing function of $u$ with $\nu_{1}(u=0)=\frac{1}{2}$ and $\nu_{1}(u \rightarrow \infty)=\frac{5}{8}$. The exponent $\nu_{3}=1 / \theta+9 \theta / 16$ decreases from $\frac{5}{2}$ to $\frac{13}{8}$ as $u$ goes from 0 to $\infty$. This result holds at any $n_{c}<1$. It agrees with the one obtained by Anderson and Ren. ${ }^{35}$

From (4.7) we obtain for the asymptotic behavior of the density-density correlation function

$$
G_{n n}(x, t) \sim n_{c}^{2}+A_{1} \frac{\cos \left(2 \mathcal{P}_{F} x+\varphi_{1}\right)}{\left|x+i v_{s} t\right|\left|x+i v_{c} t\right|^{(\theta / 4)}}+A_{2} \frac{\cos \left(4 \mathcal{P}_{F} x+\varphi_{2}\right)}{\left|x+i v_{c} t\right|^{\theta}}+A_{3} \frac{x^{2}-\left(v_{c} t\right)^{2}}{\left[x^{2}+\left(v_{c} t\right)^{2}\right]^{2}}+A_{4} \frac{x^{2}-\left(v_{s} t\right)^{2}}{\left[x^{2}+\left(v_{s} t\right)^{2}\right]^{2}}
$$

Because of the range of variation of $\theta$, the leading correction beyond the constant term is the term $\propto A_{1}$ for all finite $u$. At $u=\infty$ an alternative description of the charge dynamics of the system is possible in terms of noninteracting spinless fermions, where $\mathcal{P}_{F}$ has to be replaced by $2 \mathcal{P}_{F} .{ }^{10}$ Hence the amplitude $A_{1}$ has to vanish in this limit and the leading oscillating contribution to the correlation function is $\propto \cos \left(4 \mathcal{P}_{F} x+\varphi\right) /\left|x+i v_{c} t\right|^{2}$.
For the spin-spin correlations we find

$$
\begin{aligned}
G_{\sigma \sigma}^{\perp}(x, t) \sim & A \frac{\cos \left(2 \mathcal{P}_{F} x+\varphi\right)}{\left|x+i v_{s} t\right|\left|x+i v_{c} t\right|^{(\theta / 4)}} \\
& +B \frac{1}{\left|x+i v_{s} t\right|^{2}} \operatorname{Re}\left(\frac{x+i v_{s} t}{x-i v_{s} t}\right)
\end{aligned}
$$


(In the absence of a magnetic field the longitudinal and the transversal correlations decay with the same power asymptotically.) As $u \rightarrow \infty$, the equal time correlation functions $G_{\sigma \sigma}(x)$ decays as $x^{-3 / 2}$ at any filling $n_{c}<1$. This agrees with recent results for the infinite- $u$ Hubbard model at quarter filling ${ }^{10,11}\left(n_{c}=\frac{1}{2}\right)$ and for an infinitely strong interacting Fermi gas (at any filling less than half). ${ }^{36}$ Our results for half filling are presented in Sec. VHI below.

For the asymptotics of singlet- and triplet-pair correlators we get

$$
\begin{aligned}
G_{p}^{(0)}(x, t) \sim & A \frac{1}{\left|x+i v_{c} t\right|^{4 / \theta}\left|x+i v_{s} t\right|} \\
& +\frac{1}{\left|x+i v_{c} t\right|^{\nu}} \operatorname{Re}\left(B e^{-2 i \mathcal{P}_{F}} x \frac{x+i v_{c} t}{x-i v_{c} t}\right)
\end{aligned}
$$

with $\nu=\frac{4}{\theta}+\frac{\theta}{4}$ increasing from 2 to $\frac{5}{2}$ as $u$ goes from 0 to $\infty$ and

$$
G_{p}^{(1)}(x, t) \sim \frac{1}{\left|x+i v_{s} t\right|\left|x+i v_{c} t\right|^{(4 / \theta)}} .
$$

Hence the equal time correlations between triplet pairs decay as $x^{-\nu}$ with $\nu$ increasing from 2 to 3 with $u$.

To conclude this section on the critical exponents of the Hubbard model without external magnetic field let us emphasize again that the explicit dependence of $\theta$ $=2 \xi\left(k_{0}\right)^{2}$ on the electron density $n_{c}$ and the Coulomb repulsion $u$ can be obtained from Eq. (5.4) (see also Fig. 1). In addition, we have given $\theta$ in terms of the compressibility in Eq. (5.16).

\section{THE FERROMAGNETIC STATE}

For sufficiently large magnetic field $h \geq h_{c}$ the ground state of the Hubbard model becomes ferromagnetic with $\mathcal{M}=n_{c} / 2$, i.e., all the spins are pointing up. For all $h \leq h_{c}$ the system is critical with correlation functions decaying as powers of the distance, and we can calculate the critical exponents by the methods outlined above. Since the Coulomb interaction in the Hamiltonian (1.2) affects electrons of opposite spin only (electrons of equal spin cannot occupy the same site due to the Pauli principle), the correlations at $h=h_{c}$ are those of free fermions. However, the conformal methods produce all the leading terms present in the limit $h=h_{c}-0$ : The ones not present in a theory of free fermions have vanishing amplitude as $h \rightarrow h_{c}$.

Clearly, $h=h_{c}$ corresponds to $\lambda_{0}=0$. Now we can use the condition (2.11) to compute $h_{c}$ as a function of $u$ and the density of electrons $n_{c}$ :

$$
h_{c}=\frac{2 u}{\pi} \int_{-\pi n_{c}}^{\pi n_{c}} d k \cos k \frac{\cos k-\cos \pi n_{c}}{u^{2}+\sin ^{2} k} .
$$

Here we have used that from $(2.4) \rho_{c}(k) \equiv 1 / 2 \pi$, and hence $k_{0}=\pi n_{c}$.

Now we can calculate the dressed charge matrix from (2.13) and obtain

$$
Z=\left(\begin{array}{cc}
1 & \alpha \\
0 & 1
\end{array}\right)
$$

where

$$
\alpha=\frac{2}{\pi} \arctan \left(\frac{\sin \pi n_{c}}{u}\right)
$$

takes values between 0 and 1 as $u$ and $n_{c}$ vary (see Fig. 2).

In the following we present the results for the asymptotic behavior of correlation functions in the case $h=h_{c}$. Note that here $\mathcal{P}_{F, \uparrow}=\pi n_{c}$ and $\mathcal{P}_{F, \downarrow}=0$. The field correlator decays asymptotically as

$$
G_{\psi \psi}(x, t) \sim \frac{1}{\left|x+i v_{c} t\right|^{\nu}\left|x+i v_{s} t\right|} \operatorname{Re}\left[\left(\frac{x+i v_{s} t}{x-i v_{s} t}\right)^{1 / 2}\right]
$$

where $\nu=\frac{1}{2}(\alpha-1)^{2}$.

The leading corrections in the asymptotics of the equal time density-density and longitudinal spin-spin correlations beyond the constant term decay as the inverse square of the distance. For the transversal spin-spin correlations we obtain

$$
G_{\sigma \sigma}^{\perp}(x, t) \sim \frac{1}{\left|x+i v_{c} t\right|^{\nu}\left|x+i v_{s} t\right|^{\nu}} \operatorname{Re}\left[A e^{-i \mathcal{P}_{F, \mathrm{l}} x}\left(\frac{x+i v_{c} t}{x-i v_{c} t}\right)^{-\alpha / 2}\left(\frac{x+i v_{s} t}{x-i v_{s} t}\right)^{\alpha / 2}\right]
$$

where $\nu=\left(1+\alpha^{2}\right) / 2$. 
The correlations for singlet and triplet pairs decay as

$$
\begin{aligned}
G_{p}^{(0)}(x, t) & \sim \frac{1}{\left|x+i v_{s} t\right|^{\nu_{0}}\left|x+i v_{c} t\right|^{\nu_{0 c}}} \operatorname{Re}\left[A e^{-i \mathcal{P}_{F, t} x}\left(\frac{x+i v_{c} t}{x-i v_{c} t}\right)^{1-\alpha / 2}\left(\frac{x+i v_{s} t}{x-i v_{c} t}\right)^{\alpha / 2}\right], \\
G_{p}^{(1)}(x, t) & \sim \frac{1}{\left|x+i v_{s} t\right|^{2}\left|x+i v_{c} t\right|^{\nu_{1}}} .
\end{aligned}
$$

The exponents here are

$$
\nu_{0 s}=\frac{1}{2}\left(1+\alpha^{2}\right), \quad \nu_{0 c}=\frac{1}{2}+2\left(1-\frac{\alpha}{2}\right)^{2}, \quad \nu_{1}=2(1-\alpha)^{2} .
$$

The dependence of $\alpha$ on the Coulomb parameter $u$ and the density of electrons $n_{c}$ is given in Eq. (6.3).

For intermediate magnetic fields $0<h<h_{c}$ the dressed charge matrix will interpolate between the expressions (5.3) and (6.2). That means that in the general case the integral equations (2.13) cannot be reduced to scalar ones and all the elements of $Z$ are nonzero (this can be seen, e.g., by considering the case of $0<h_{c}-h \ll h_{c}$ and $n_{c}$ close to 0 or 1 when both $\lambda_{0} \ll 1$ and $\sin k_{0} \ll 1$ ). We discuss the dependence of the dressed charge on an external magnetic field in detail for the case of half filling below.

\section{HALF-FILLED BAND}

\section{A. The dressed charge at half filling}

Half filling, i.e., $n_{c}=1$, corresponds to $k_{0}=\pi$. It is well known that there exists a gap in the spectrum of charge excitations in this case. The contributions of the spin degrees of freedom to the finite-size scaling behavior have been found to be equivalent to those of the onedimensional isotropic Heisenberg model. ${ }^{6}$ The sets of integral equations (2.4), and (2.9), can be reduced to scalar ones for the spin components only:

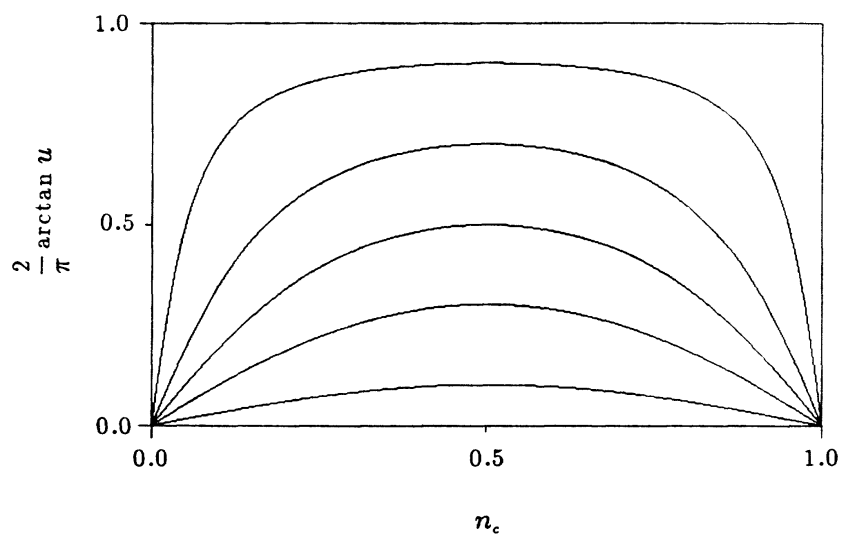

FIG. 2. Lines of constant universal behavior at $h=h_{c}$. Shown are contours of constant $\alpha$ [Eq. (6.4)] in the $n_{c^{-}} u$ plane: $\alpha \rightarrow 1(0)$ as $u \rightarrow 0(\infty)$.

$$
\begin{aligned}
& \rho_{s}(\lambda)=\rho_{s}^{(0)}(\lambda)-\frac{1}{2 \pi} \int_{-\lambda_{0}}^{\lambda_{0}} d \mu K_{2}(\lambda-\mu) \rho_{s}(\mu) \\
& \varepsilon_{s}(\lambda)=\varepsilon_{s}^{(0)}(\lambda)-\frac{1}{2 \pi} \int_{-\lambda_{0}}^{\lambda_{0}} d \mu K_{2}(\lambda-\mu) \varepsilon(\mu)
\end{aligned}
$$

The bare density and energy are given by (since the number of electrons is fixed we can put the chemical potential to zero)

$$
\begin{gathered}
\rho_{s}^{(0)}(\lambda)=\frac{1}{(2 \pi)^{2}} \int_{-\pi}^{\pi} d k K_{1}(\sin k-\lambda) \\
\varepsilon_{s}^{(0)}(\lambda)=h-\frac{1}{2 \pi} \int_{-\pi}^{\pi} d k \cos ^{2} k K_{1}(\sin k-\lambda) .
\end{gathered}
$$

The density and energy of the charge components can be given in terms of the solution of (7.1):

$\rho_{c}(k)=\frac{1}{2 \pi}+\frac{\cos k}{2 \pi} \int_{-\lambda_{0}}^{\lambda_{0}} d \lambda K_{1}(\sin k-\lambda) \rho_{s}(\lambda)$,

$\varepsilon_{c}(k)=-\frac{h}{2}-2 \cos k+\frac{1}{2 \pi} \int_{-\lambda_{0}}^{\lambda_{0}} d \lambda K_{1}(\sin k-\lambda) \varepsilon_{s}(\lambda)$

The critical behavior of the theory is again determined by a dressed charge, which is the solution to the following equation:

$$
\xi_{s}(\lambda)=1-\frac{1}{2 \pi} \int_{-\lambda_{0}}^{\lambda_{0}} d \mu K_{2}(\lambda-\mu) \xi_{s}(\mu) .
$$

[This is, in fact, just the equation for $\xi_{s s}$ in (2.13) for $k_{0}=\pi$.]

As mentioned above, the spin part of the spectrum is still massless and can be described by a Gaussian field theory with central charge $c=1$ and operator dimensions given by Eq. (3.4):

$$
\Delta^{ \pm}=\frac{1}{2}\left(D_{s} \xi_{s}\left(\lambda_{0}\right) \pm \frac{\Delta N_{s}}{2 \xi_{s}\left(\lambda_{0}\right)}\right)^{2} .
$$

For given value of $u$ the boundaries of the integrals in Eqs. (7.1) and (7.4) depend on the magnetic field. From Eq. (2.11) we find that the Fermi sea of spin waves collapses for $h \geq h_{c}=2\left(\sqrt{u^{2}+1}-u\right)$, i.e., $\lambda_{0}=0$, and the 
ground state of the system becomes ferromagnetic. As the magnetic field approaches $h_{c}$ from below we have

$$
\lambda_{0}=\left(u^{2}+1\right)^{3 / 4} \sqrt{h_{c}-h} .
$$

The system is critical for $h \leq h_{c}$, the critical exponents are functions of the dressed charge which can be obtained from (7.4) to be

$$
\xi_{s}\left(\lambda_{0}\right)=1-\frac{\left(u^{2}+1\right)^{3 / 4}}{\pi u} \sqrt{h_{c}-h}
$$

for magnetic fields close to $h_{c}$.

As used before, for vanishing magnetic field we have $\lambda_{0} \rightarrow \infty$. For small magnetic field $\lambda_{0}$ depends on $h \log -$ arithmically,

$$
\lambda_{0}=\frac{2 u}{\pi} \ln \left(h_{0} / h\right) .
$$

Using the Wiener-Hopf method, $h_{0}$ can be found from applying the condition (2.11) to the solution of Eq. (7.1) for the dressed energy to be ( $I_{1}$ is a modified Bessel function)

$$
h_{0}=4 I_{1}\left(\frac{\pi}{2 u}\right)\left(\frac{2 \pi}{e}\right)^{1 / 2}
$$

Again we can compute the dependence of the dressed charge $\xi_{s}\left(\lambda_{0}\right)$ on a small magnetic field (i.e., large $\left.\lambda_{0}\right)$ perturbatively from Eq. (7.4). As for the isotropic Heisenberg antiferromagnet ${ }^{20}$ we find

$$
\xi_{s}\left(\lambda_{0}\right)=\frac{1}{\sqrt{2}}\left(1+\frac{1}{4 \ln \left(h_{0} / h\right)}\right) .
$$

The dependence of $h_{0}$ on $u$ is given by (7.9). Note that for $h=0$ the coupling constant $\xi_{s}\left(\lambda_{0}\right)$ of the Gaussian theory is fixed to the self-dual point of this model, namely the $k=1 \mathrm{SU}(2)$ Wess-Zumino-Witten model, which is the only possible choice compatible with the spin- $\frac{1}{2}$ symmetry of this system.

Before we present our results for the correlation functions, let us first express $\xi\left(\lambda_{0}\right)$ in terms of a thermodynamical exponent, in this case the magnetic susceptibility

$$
\chi(h)=\frac{\partial \mathcal{M}}{\partial h} .
$$

To do so we consider the change in the ground-state energy of the system due to a change in the magnetization $\Delta \mathcal{M}=-\Delta N_{s} / N$. Comparison of the result from the finite-size scaling analysis (3.2)

$$
\Delta E=\frac{\pi v_{s}}{2 N} \frac{1}{\left[\xi_{s}\left(\lambda_{0}\right)\right]^{2}}\left(\Delta N_{s}\right)^{2}
$$

with the one obtained from an expansion of $\Delta E=\Delta(\tilde{E}$ $-N h \mathcal{M})$ with respect to $\Delta \mathcal{M}$ we find

$$
\xi_{s}\left(\lambda_{0}\right)^{2}=\pi v_{s} \chi(h) \text {. }
$$

(Here we have used that $\partial \tilde{E} / \partial \mathcal{M}=h N$.) Again this relation is equivalent to the one found in the isotropic Heisenberg antiferromagnet. ${ }^{20}$

\section{B. Correlation functions at half filling}

As mentioned above, only the density-density (4.2) and spin-spin correlation functions (4.3) decay as a power of the distance for the Hubbard model with half-filled band. The asymptotic behavior of these can be found by the methods outlined above using the result (3.3) for the correlation functions of operators in a conformally invariant theory. Note that the momentum of the intermediate state is now given by $2 D_{s} \mathcal{P}_{F, \downarrow}$ for states with $\Delta N_{s}$ even but $\pi+2 D_{s} \mathcal{P}_{F, \downarrow}$ for states with $\Delta N_{s}$ odd. Again we will write down the critical exponents in terms of $\theta=2 \xi_{s}\left(\lambda_{0}\right)^{2}$ which grows from 1 for vanishing magnetic field to 2 for $h=h_{c}$. For the density-density correlations we obtain

$$
\begin{aligned}
G_{n n}(x, t) \sim & 1+A_{1} \frac{\cos \left(2 \mathcal{P}_{F, \downarrow} x+\varphi\right)}{\left|x+i v_{s} t\right|^{\theta}} \\
& +A_{2} \frac{x^{2}-\left(v_{s} t\right)^{2}}{\left[x^{2}+\left(v_{s} t\right)^{2}\right]^{2}}
\end{aligned}
$$

Similarly, we obtain for the spin-spin correlation functions

$$
\begin{aligned}
G_{\sigma \sigma}^{\perp}(x, t) \sim & A_{1} \frac{\cos \left(\pi x+\varphi_{1}\right)}{\left|x+i v_{s} t\right|^{(1 / \theta)}} \\
& +\frac{1}{\left|x+i v_{s} t\right|^{\nu}} \operatorname{Re}\left(A_{2} e^{-i\left(\pi+2 \mathcal{P}_{F, \downarrow}\right) x} \frac{x+i v_{s} t}{x-i v_{s} t}\right)
\end{aligned}
$$

$(\nu=\theta+1 / \theta) ; G_{\sigma \sigma}^{z}$ is identical to (7.14) with the constant term replaced by $\mathcal{M}^{2}$. At $h=0$ we have $\theta=1, \nu=2$. Hence, the spin correlations are isotropic and decay as $A_{1} \cos (\pi x) / x$ at equal times. As mentioned above, deviations from conformal fields lead to logarithmic corrections to these expressions. For example, the coefficient $A_{1}$ in the equal time spin-spin correlation functions at $h=0$ has been found to be ${ }^{9}$

$$
A_{1} \propto \sqrt{\ln |x|} .
$$

(The same result has been obtained for the isotropic Heisenberg antiferromagnet in Ref. 33.)

Let us stress again that $\theta$ is a function of the magnetic field, the exact dependence being given by Eq. (7.4).

\section{PHYSICAL MEANING OF THE DRESSED CHARGE MATRIX}

In the previous sections we have shown the importance of the dressed charge matrix $Z$ (3.9) for the description of the critical behavior of the Hubbard model. In addition we have obtained relations for the dressed charge that allow for a physical interpretation in several limiting (scalar) cases (5.12), (5.16), and (7.13). In this section we want to generalize these relations to the case with two degrees of freedom (i.e., spin and charge). First of all it is straightforward to generalize (5.12) to a matrix situation: 8,30

$$
\frac{1}{2 \rho_{\beta}\left(q_{\beta}\right)} \frac{\partial n_{\alpha}}{\partial q_{\beta}}=Z_{\alpha \beta}
$$


where $\alpha$ and $\beta$ take values $c, s$ and $q_{c}=k_{0}, q_{s}=\lambda_{0}$. Again, the dressed charge matrix $Z$ governs the characteristic changes of the distribution functions for charges and spin waves at the Fermi surface due to changes of the density and magnetization.

Now let us try to find a generalization of Eqs. (5.16) and (7.13) where the dressed charge was related to the compressibility and the magnetic susceptibility, respectively, to the general case: Again we want to express the finite-size corrections (3.6) with $D_{c}=D_{s}=0$,

$$
\Delta E=\frac{\pi}{2 N} \Delta \mathrm{N}^{T}\left(Z^{-1}\right)^{T} V Z^{-1} \Delta \mathrm{N}
$$

in terms of thermodynamic coefficients. To do so we proceed as in Sec. V A and expand

$$
E=\tilde{E}+\mu N_{c}-N h \mathcal{M}
$$

in small changes of the number of charges $N_{c}$ and the magnetization $\mathcal{M}$. To second order this gives

$$
\begin{aligned}
\Delta E= & \left(\frac{\partial \tilde{E}}{\partial N_{c}}+\mu\right) \Delta N_{c}+\left(\frac{\partial \tilde{E}}{\partial \mathcal{M}}-N h\right) \Delta \mathcal{M} \\
& +\frac{1}{2} \frac{\partial^{2} \tilde{E}}{\partial N_{c}^{2}}\left(\Delta N_{c}\right)^{2}+\frac{1}{2} \frac{\partial^{2} \tilde{E}}{\partial \mathcal{M}^{2}}(\Delta \mathcal{M})^{2} \\
& +\frac{\partial^{2} \tilde{E}}{\partial N_{c} \partial \mathcal{M}} \Delta N_{c} \Delta \mathcal{M} .
\end{aligned}
$$

Using $\Delta \mathcal{M}=\left(\Delta N_{c} / 2 N-\Delta N_{s} / N\right)$ this can be compared to Eq. (8.2) to obtain

$$
\frac{\partial \tilde{E}}{\partial N_{c}}=-\mu, \quad \frac{\partial \tilde{E}}{\partial \mathcal{M}}=N h,
$$

and the desired relation for the dressed charge matrix (3.9):

$$
\pi\left(Z^{-1}\right)^{T} V Z^{-1}=\left(\begin{array}{cc}
\frac{1}{n_{c}^{2} \kappa}+\frac{1}{4 \chi}+\frac{1}{\eta} & -\frac{1}{2 \chi}-\frac{1}{\eta} \\
-\frac{1}{2 \chi}-\frac{1}{\eta} & \frac{1}{\chi}
\end{array}\right)
$$

Here the compressibility $\kappa$ and the susceptibility $\chi$ have been given before, while

$$
\eta=\frac{1}{N} \frac{\partial N_{c}}{\partial h}=\frac{\partial \mathcal{M}}{\partial \mu} .
$$

Note that (8.6) almost solves the problem. Since the matrix $Z$ enters in a symmetric combination of $Z^{-1}$ and $\left(Z^{-1}\right)^{T}$, however, we have found just three equations to fix the four elements of $Z$.
Following the ideas of Ref. 37 we can obtain a fourth equation: Let us change the boundary conditions leading to Eqs. (2.1) by adding a constant phase $\varphi$ to the equations for the momenta $k_{j}$ of the charges

$$
N k_{j}=2 \pi I_{j}+\varphi+\sum_{\beta=1}^{N_{s}} 2 \arctan \left(\frac{\sin k_{j}-\lambda_{\beta}}{u}\right),
$$

while leaving the equations for the rapidities of spin waves unchanged. Physically this additional phase can be obtained by enclosing a magnetic flux in the ring on which the electrons can move. For small $\varphi$ this leads to a change in the ground-state energy $\Delta E \propto \varphi^{2}$. The momentum of this state will be

$$
P=n_{c} \varphi \text {. }
$$

This is the Aharonov-Bohm effect for this system. The current is given as $j(\varphi)=\partial E(\varphi) / \partial \varphi$.

On the other hand this change in boundary conditions corresponds to the finite-size corrections (3.6) for an excited state with $\Delta N_{c}=\Delta N_{s}=D_{s}=0$ and $D_{c}=\varphi / 2 \pi$. Hence the change in energy can be given in terms of elements of the dressed charge matrix as

$$
\Delta E(\varphi)=\frac{1}{2 \pi N}\left(v_{c} Z_{c c}^{2}+v_{s} Z_{c s}^{2}\right) \varphi^{2}
$$

This is also related to an observable quantity.

To conclude we have derived a set of equations, (8.6) and $(8.10)$, that relates the elements of the dressed charge matrix to observable quantities such as thermodynamic coefficients and velocities of spin waves and charge-density waves. Hence, the universal critical behavior of the 1D Hubbard chain can be determined by taking certain combinations of these quantities. We believe that these relations continue to hold for interacting one-dimensional electron systems even away from the integrable point (1.2).

We have recently found that some of our results for correlation functions in the absence of a magnetic field have been obtained independently by several other authors. ${ }^{38-41}$

\section{ACKNOWLEDGMENTS}

We wish to thank Professor P. W. Anderson and Y. Ren for a discussion on this topic. One of the authors (H.F.) gratefully acknowledges financial support by the Deutsche Forschungsgemeinschaft and by the National Science Foundation (NSF) under Grant No. DMR8810541. Additional support was provided by the NSF under Grant No. PHY-89-08495.

\footnotetext{
${ }^{1}$ E. H. Lieb and F. Y. Wu, Phys. Rev. Lett. 20, 1445 (1968).

${ }^{2}$ M. Takahashi, Prog. Theor. Phys. 47, 69 (1972).

${ }^{3}$ F. Woynarovich, J. Phys. C 15, 85 (1982); 15, 97 (1982); 16, 5293 (1983).
}

${ }^{4}$ B. Sutherland, in Exactly Solvable Problems in Condensed Matter and Relativistic Field Theory, Vol. 242 of Lecture Notes in Physics, edited by B. S. Shastry, S. S. Jha, and V. Singh (Springer-Verlag, Berlin, 1985), p. 1. 
${ }^{5}$ B. S. Shastry, Phys. Rev. Lett. 56, 2453 (1986).

${ }^{6}$ F. Woynarovich and H.-P. Eckle, J. Phys. A 20, L443 (1987).

${ }^{7}$ N. M. Bogoliubov and V. E. Korepin, Mod. Phys. Lett. B 1, 349 (1988); Int. J. Mod. Phys. B 3, 427 (1989).

${ }^{8}$ F. Woynarovich, J. Phys. A 22, 4243 (1989).

${ }^{9}$ A. M. Finkel'shtein, Pis'ma Zh. Eksp. Teor. Fiz. 25, 83 (1977) [JETP Lett. 25, 73 (1977)].

${ }^{10}$ M. Ogata and H. Shiba, Phys. Rev. B 41, 2326 (1990).

${ }^{11}$ A. Parola and S. Sorella, Phys. Rev. Lett. 64, 1831 (1990).

${ }^{12}$ A. Luther and I. Peschel, Phys. Rev. B 12, 3906 (1975).

${ }^{13}$ K. B. Efetov and A. I. Larkin, Zh. Eksp. Teor. Fiz. 66, 2290 (1974) [Sov. Phys. JETP 39, 1129 (1974)].

${ }^{14}$ V. N. Popov, Pis'ma Zh. Eksp. Teor. Fiz. 31, 560 (1980) [JETP Lett. 31, 526 (1980)].

${ }^{15}$ F. D. M. Haldane, Phys. Rev. Lett. 45, 1358 (1980); J. Phys. C 14, 2589 (1981).

${ }^{16}$ F. D. M. Haldane, Phys. Lett. 81A, 153 (1981).

${ }^{17}$ F. D. M. Haldane, Phys. Rev. Lett. 47, 1840 (1981).

${ }^{18}$ F. D. M. Haldane, in Electron Correlation and Magnetism in Narrow-Band Systems, edited by T. Moriya (SpringerVerlag, Berlin, 1981), p. 150.

${ }^{19}$ L. Kadanoff and A. C. Brown, Ann. Phys. (N. Y.) 121, 318 (1979).

${ }^{20}$ N. M. Bogoliubov, A. G. Izergin, and V. E. Korepin, Nucl. Phys. B275 [FS17], 687 (1986).

${ }^{21}$ A. A. Belavin, A. M. Polyakov, and A. B. Zamolodchikov, Nucl. Phys. B241, 333 (1984).

${ }^{22}$ J. L. Cardy, Nucl. Phys. B270 [FS16], 186 (1986).

${ }^{23}$ H. W. J. Blöte, J. L. Cardy, and M. P. Nightingale, Phys.
Rev. Lett. 56, 742 (1986); I. Affleck, ibid. 56, 746 (1986).

${ }^{24}$ H. J. de Vega and F. Woynarovich, Nucl. Phys. B251, 439 (1985); F. Woynarovich and H.-P. Eckle, J. Phys. A 20, L97 (1987).

${ }^{25}$ N. M. Bogoliubov, A. G. Izergin, and N. Yu. Reshetikhin, J. Phys. A 20, 5361 (1987).

${ }^{26}$ S. V. Pokrovskii and A. M. Tsvelick, Zh. Eksp. Teor. Fiz. 93, 2232 (1987) [Sov. Phys. JETP 66, 1275 (1987)].

${ }^{27}$ H. J. de Vega, J. Phys. A 20, 6023 (1987).

${ }^{28} \mathrm{~A}$. Berkovich and G. Murthy, Phys. Lett. A 142, 121 (1989).

${ }^{29}$ A. G. Izergin, V. E. Korepin, and N. Yu. Reshetikhin, J. Phys. A 22, 2615 (1989).

${ }^{30}$ H. Frahm and N.-C. Yu, J. Phys. A 23, 2115 (1990).

${ }^{31}$ G. Albertini and B. M. McCoy (unpublished).

${ }^{32}$ C. N. Yang, Phys. Rev. Lett. 19, 1312 (1967).

${ }^{33}$ I. Affleck, D. Gepner, H. J. Schulz, and T. Ziman, J. Phys. A 22, 511 (1989).

${ }^{34}$ C. N. Yang and C. P. Yang, Phys. Rev. 150, 327 (1966).

${ }^{35} \mathrm{P}$. W. Anderson and Y. Ren (private communication).

${ }^{36}$ A. A. Ovchinnikov and A. V. Zabrodin, Phys. Lett. A 138, 139 (1989).

${ }^{37}$ B. Sutherland and B. S. Shastry (unpublished); B. S. Shastry and B. Sutherland, Phys. Rev. Lett. 65, 243 (1990).

${ }^{38} \mathrm{M}$. Brech, J. Voit, and H. Büttner, Europhys. Lett. 12, 289 (1990).

${ }^{39}$ H. J. Schulz, Phys. Rev. Lett. 64, 2831 (1990).

${ }^{40}$ N. Kawakami and S.-K. Yang, Phys. Lett. A 148, 359 (1990).

${ }^{41}$ A. M. Tsvelick (unpublished). 